\title{
Liebe Leserinnen, liebe Leser!
}

Vor Ihnen liegt eine etwas andere e\&j-Ausgabe - ein Sonderheft, das aus Anlass des 120jährigen Bestehens unseres Verbandes entstanden ist.

Der Österreichische Verband für Elektrotechnik OVE - 1883 als Elektrotechnischer Verein in Wien gegründet - hat die österreichische Industrie und die Berufsgruppe der Elektrotechniker in den 120 Jahren seines Bestehens über viele turbulente Zeiten hinweg begleitet. Die Gründungsziele des OVE am Ende des 19. Jahrhunderts ergaben sich aus dem enormen Aufstreben von elektrotechnischen Anwendungen und den dadurch bedingten Problemen in der Handhabung

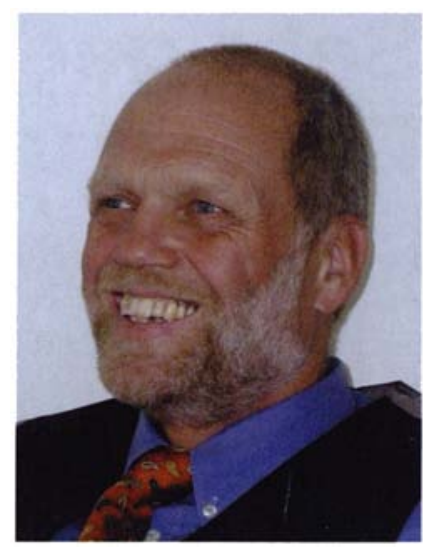
dieser neuen Technik:

Im Vordergrund stand die Sicherheit der elektrotechnischen Anwendungen. Dem jungen Verband war es ein Anliegen, dem Techniker die Verantwortung für seine Produkte bewusst zu machen. Gleichzeitig galt es, das Wissen rund um die Elektrotechnik zu sammeln und auch weiterzugeben. Aus diesen Zielen heraus entwickelten sich auch die Geschäftsfelder des OVE - die elektrotechnische Normung, Zertifizierung und die fachgerechte Aus- und Weiterbildung.

Wir wollen mit diesem Sonderheft nicht zurückblicken - dazu steht allen Interessierten ein Archiv aus 120 Jahrgängen unserer Zeitschrift zur Verfügung, welches ein unvergleichliches und wertvolles Spiegelbild der Leistungen der österreichischen Wissenschaft und Industrie abgibt. Vielmehr erfolgt mit dieser e\&i-Ausgabe ein bewusster Blick nach vor, in eine interessante und spannende Zukunft unserer Branche.

Dieses Sonderheft trägt den bedeutenden Titel „Visionen der Elektrotechnik und Informationstechnik“. Vertreter aus Lehre, Wissenschaft, Forschung und aus der Industrie haben in diesem Heft ihre jeweilige (Branchen-)Vision dargelegt. Das Ergebnis bietet eine fulminante Bandbreite, wie sich die Zukunft der Elektrotechnik und Informationstechnik gestalten lässt!

Nicht nur in dieser e\&i-Ausgabe, sondern auch im OVE selbst, gibt es Visionen. Im Rahmen eines intensiv geführten Strategieprozesses, zu dem auch alle Partner des OVE eingeladen waren, wurde die aktuelle Positionierung unseres Verbandes durchleuchtet. Ebenso wurde analysiert, wie sich der OVE den künftigen Herausforderungen stellen kann. In dieser Strategiediskussion hat sich bestätigt, dass die Gründungsziele unseres Verbandes nach wie vor Gültigkeit besitzen. Das Ergebnis des Strategieprozesses finden Sie in nachfolgenden Kernaussagen dargelegt:

- Der OVE versteht sich als kompetente, unabhängige Plattform in allen Fragen und Bereichen der Elektrotechnik und Informationstechnik für seine Mitglieder und den gesamten Berufsstand.

- Der OVE ist anerkannte Autorität für Sicherheit (Safety und Security), im Besonderen auf dem Gebiet der Elektrotechnik und Informationstechnik.

- Der OVE agiert flexibel und passt seine Dienstleistungen laufend geänderten Rahmenbedingungen des Marktes an. Dies gilt nicht nur für bestehende Branchensegmente, sondern betrifft auch die Forcierung von Schlüsseltechnologien in neu entstehenden Segmenten des Brachenumfelds.

- In den Gesellschaften des OVE finden alle Sparten und Berufsgruppen der Branche eine Heimat. Die Mitglieder werden mit Networking, Informations- und Weiterbildungsveranstaltungen und weiteren Serviceleistungen unterstützt.

Wesentlich und für das Agieren des OVE förderlich ist, dass unsere Partnerverbände ebenso wie die Behörde und auch die Wissenschaft voll hinter dem OVE stehen. Mit anderen Worten und auf den Punkt gebracht: Der OVE ist anerkannter Vertreter unserer Branche.

Die Elektrotechnik leitete zusammen mit der Chemie den 3. Kondratieff-Zyklus ein, der von 1900 bis 1950 das wirtschaftliche Geschehen positiv beeinflusste. Die Entwicklungen auf Basis der Elektrotechnik haben selbstredend auch dem 5. Kondratieff-Zyklus Informationstechnik - einen kräftigen Impuls versetzen können. Dass diese mit ihren weiten Feldern auch den nächsten - das wäre der 6. Kondratieff-Zyklus - einleiten können, wäre wünschenswert.

Die Visionen des OVE sind zahlreich und werden bereits von maßgeblichen Vertretern unserer Mitgliedsfirmen geteilt. Nun gilt es, diese in den kommenden Jahren umzusetzen.

Folgend finden Sie Grüße unseres befreundeten Verbandes in Deutschland, dem VDE, für die wir uns sehr herzlich bedanken!

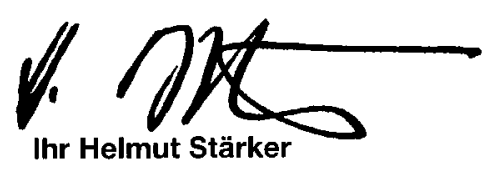

